\title{
UTILIZACIÓN DE FAUNA SILVESTRE EN EL ÁREA RURAL DE CAIMITO, SUCRE, COLOMBIA
}

\author{
WILDLIFE USE IN THE RURAL AREA OF CAIMITO, SUCRE, COLOMBIA \\ DE LA OSSA-LACAYO, ALEJANDRO ${ }^{1}$ Ecólogo, DE LA OSSA, V. JAIME² Dr. \\ ${ }^{1}$ Pontificia Universidad Javeriana, Maestría en Desarrollo Rural, Colombia. \\ ${ }^{2}$ Universidad de Sucre, Facultad de Ciencias Agropecuarias, Grupo de \\ Investigación en Biodiversidad Tropical, Colombia.
}

*Correspondencia: alejandrodelaossa@yahoo.com

Recibido: 22-03-2012; Aceptado: 13-04-2012.

\section{Resumen}

Esta investigación analiza la caza de subsistencia y registra las especies de reptiles, aves y mamíferos que son capturados para subsistencia o comercio en una zona rural del municipio de Caimito, Sucre, Colombia. La zona se caracteriza por la presencia de extensas ciénagas y forma parte de la región de la Mojana en el San Jorge. Se aplicaron entrevistas semiestructuradas a habitantes rurales, conocedores y usuarios del recurso. Se evidencia que las especies de mayor preferencia, caracterizadas por un mayor valor social, son aquellas que de forma particular están asociadas a la tradición cultural de consumo. Los registros permiten conocer preliminarmente el estado de la fauna silvestre que se está utilizando y permitirá proponer futuros estudios ambientales que conduzcan a proyectos de conservación en la zona.

Palabras clave: fauna silvestre, cacería de subsistencia, Caimito, Sucre.

\section{Abstract}

This research analyzed the subsistence hunt and registered the species of reptiles, birds and mammals that are captured for consumption or trade in a rural zone of Caimito municipality, Sucre, Colombia. The zone is characterized by the presence of extensive marshes and it is part of the region of the Mojana at the San Jorge river basin. Semistructured interviews were applied to the rural inhabitants, experts and users of the resource. It is evidenced that the species of more preference, are characterized by a bigger social use value and are those that are associated to the tradition of cultural consumption in a particular way. In essence the registrations allow introductorily knowing the state of the wildlife that is using to propose future environmental studies that lead to conservation projects.

Key words: wildlife, use, subsistence hunting, Sucre.

\section{Introducción}

La fauna silvestre es un componente ecosistémico de primordial importancia para la sociedad, fue y sigue siendo utilizada como fuente de alimentación y 
para diversa manufactura (REDFORD y ROBINSON, 1991; OJASTI, 2000; ROBINSON y BENNETT, 2000). Forma parte del acervo cultural y artístico de muchos pueblos (STEARMAN, 2000). Algunas especies son de gran aprecio como mascotas (REDFORD y ROBINSON, 1991).

Como lo aseveran DE LA OSSA (1998) y BAPTISTE-BALLERA et al, (2002) su utilización es antigua y se relaciona con la historia del hombre, se registra como fundamental parte de su dieta y de histórica influencia en el desarrollo cultural de la humanidad. BAPTISTE-BALLERA et al. (2002) indican que la importancia económica de la fauna no es fácilmente evaluable a nivel de su comercio internacional y que regional y localmente es aún más difícil de dilucidar porque entran en juego las diferentes características de los grupos humanos, la disponibilidad relativa ante otros recursos y el contexto sociopolítico y simbólico regional, además de los aspectos legales y normativos sobre uso y la paradoja entre cultura, necesidad de subsistencia y paradigmas de la conservación, que se entrelazan en una mezcla que favorece el consumo ilegal y propician la ilegalidad (OJASTI, 2000).

Es evidente que los cambios en el modo de producción dentro de todas las comunidades o grupos humanos no se han dado en forma simultánea, lo cual es inequitativo y se manifiesta en la existencia de una gran variedad de formas de producción, entre las que se pueden encontrar aún y como fue hace cientos de años atrás, etnias o grupos humanos cuya subsistencia se basa en la recolección y la cacería, mientras otros un poco más avanzados relacionan la agricultura y la cacería, teniendo en común la explotación para subsistencia de la fauna silvestre ((REDFORD y ROBINSON, 1991; OJASTI, 2000; ROBINSON y BENNETT, 2000; DE LA OSSA y DE LA OSSA-LACAYO, 2011).

Entre las especies de fauna silvestre de mayor importancia comercial y de consumo en Colombia, están: Hydrochaeris hydrochaeris (ponche, chigüiro), Agouti paca (guartinaja, boruga, paca), Tayassu sp. (zaino, puerco de monte), Dasyprocta sp (ñeque, picure), Odocoileus virginianus (venado), Mazama sp (venado soche), Dasypus novencinctus (armadillo), Silvilagus floridanus (conejo), Iguana iguana (iguana), Trachemys callirostris (hicotea) (PÉREZ y OJASTI, 1996; RAMÍREZ-PERILLA, 1996; AGUILERA-DÍAZ, 2004), las cuales también son de gran importancia en la zona de la Mojana (DE LA OSSA y DE LA OSSA-LACAYO, 2011). En general, el consumo de carne de animales silvestres resulta ser el uso más importante dado a este componente en todo el país, incluso en algunas regiones en donde existe la más alta oferta de ganado vacuno (POLANCO, 2000; DE LA OSSA y DE LA OSSA-LACAYO, 2011). 
El municipio de Caimito, Sucre, por su ubicación geográfica y por razones de índole cultural ha sido uno de los centros de explotación de fauna silvestre, que existen en la región de la depresión momposina, en la denominada Mojana del bajo San Jorge, con raíces sociales y culturales muy profundas (DE LA OSSA, 2003; AGUILERA-DÍAZ, 2004). El conocimiento que tienen los pescadores o cazadores locales de esta zona, permite abordar con mayor objetividad los procesos de aprovechamiento de la fauna silvestre en un contexto consuntivo, que puede servir como base para aplicar políticas integrales de conservación sin desconocer la realidad social, económica y cultural circundante (ALDANA et al., 2006; DE LA OSSA y DE LA OSSA-LACAYO, 2011).

Esta investigación pretende estimar la importancia de los reptiles, aves y mamíferos silvestres reconocidos y utilizados por las comunidades rurales del municipio de Caimito en el departamento de Sucre, Colombia. Sus resultados son parte de un proyecto regional que evalúa los patrones de utilización de fauna silvestre para la zona sur del departamento de Sucre, mediante el cual se busca evaluar el proceso extractivista con fines de subsistencia dentro de un entorno que se cataloga como marginal tanto económica como ambientalmente..

\section{Materiales y métodos}

Caracterización del área de estudio: el municipio de Caimito (Fig. 1) está situado en el suroeste del departamento de Sucre a $8^{\circ} 47^{\prime} \mathrm{N}$ y $75^{\circ} 23^{\prime} \mathrm{O}$, en la denominada región de la Mojana, depresión Momposina, bajo río San Jorge, Colombia. Este sistema ambiental actúa como regulador de los ríos Magdalena, Cauca y San Jorge; mayoritariamente la zona está conformada por humedales (AGUILERA-DÍAZ, 2004); además de poseer importantes y variados hábitat para la fauna silvestre regional y ser por excelencia un lugar de tradicional extractivismo faunístico (DE LA OSSA, 2003). La zona está conformada por llanuras fluvio deltaicas y fluvio lacustres; con zonas típicamente de bosque seco tropical a bosque muy seco tropical y pequeños enclaves de sabanas naturales (AGUILERA-DÍAZ, 2005).

Muestreos: para la identificación de los usos de la fauna se realizaron en la

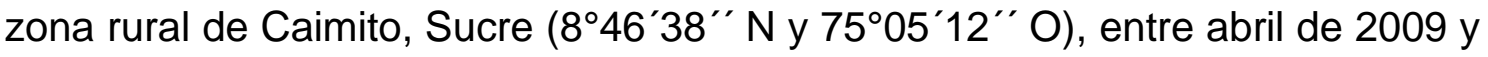
marzo de 2010, un total de cuatro sesiones de trabajo, equivalentes a una sesión cada tres meses. Se aplicaron un total de 80 entrevistas semiestructuradas a pescadores y/o extractivistas previamente identificados, procedentes de la zona inundable, con permanencia en la zona de por lo menos 20 años (MARGOLUIS y SALAFSKY, 1998); la muestra cubrió el 64\% de los extractivistas activos. Se llevó a cabo una comprobación de imágenes 
para reconocimiento de cada especie registrada en las encuestas por parte del entrevistado (HILTY y BROWN, 1986; EMMONS, 1991; RENGIFO y LUNDBERG, 1999).

Análisis de la información: Los usos se clasificaron en siete categorías: carne (consumo o venta) (CCV), mascotas (tenencia en casa) (MC), huevos (consumo o venta) (HCV), crías (venta) (CV), piel (venta) (PV), medicinal (M) y daños (sembrados o producción pecuaria) (D). El análisis de la información fue porcentual y se aplicó prueba de chi cuadrado para estimar la significancia o importancia de cada taxón, cada especie y su uso.

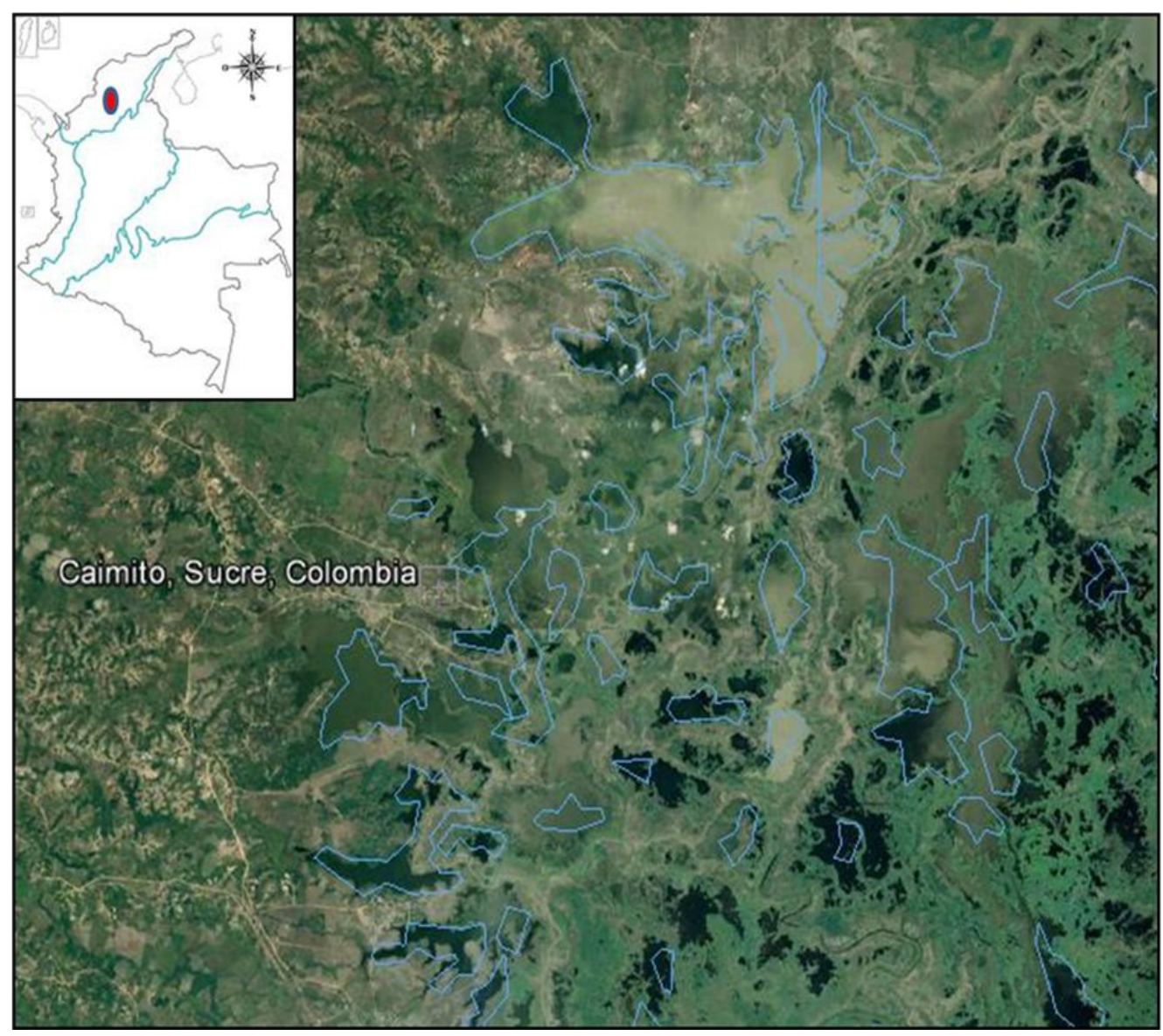

Figura 1. Área de estudio (Google Earth, versión 5.0)

\section{Resultados}

En total se registró el uso de 42 especies (Tabla 1) de las cuales fueron 6 especies de reptiles (14,3\%), 28 especies de aves (66,7\%) y 8 especies de mamíferos (19,0\%). Los usos generales dados, fueron: (CCV) carne consumo o venta $23(54,6 \%)$, (MC) tenencia de mascotas $10(23,8 \%)$, (HCV) consumo o venta de huevos 8 (19,0\%), (CV) venta de crías 20 (47,6\%), (PV) venta de piel 
$3(7,1 \%)$, (M) medicinal 12 (28,6\%) y (D) daños sembrados o producción 11 $(26,2 \%)$. En la Tabla 2 se presentan los datos porcentuales de cada uno de los usos detectados de acuerdo con el taxón.

Tabla 1. Uso de reptiles, aves y mamíferos detectados en la zona de estudio. $R$ $=$ Reptil, $\mathrm{A}=$ Ave, $\mathrm{M}=$ Mamífero, To = Total, CCV =Carne (consumo o venta), $\mathrm{MC}=$ mascotas (tenencia en casa), $\mathrm{HCV}=$ huevos (consumo o venta), $\mathrm{CV}=$ crías (venta), $\mathrm{PV}=$ piel (venta), $\mathrm{M}=$ medicinal, $\mathrm{D}=$ daños (sembrados 0 producción pecuaria)

\begin{tabular}{|c|c|c|c|c|c|c|c|c|c|c|}
\hline Especie & N. Común & Taxón & $\begin{array}{c}\text { CCV } \\
23\end{array}$ & \begin{tabular}{c|}
$M C$ \\
10
\end{tabular} & $\begin{array}{c}\text { HCV } \\
8\end{array}$ & \begin{tabular}{|l|} 
CV \\
20
\end{tabular} & \begin{tabular}{|c|} 
PV \\
$\mathbf{3}$
\end{tabular} & \begin{tabular}{|l|}
$M$ \\
12
\end{tabular} & \begin{tabular}{|c|}
$D$ \\
11
\end{tabular} & To \\
\hline Iguana iguana & Iguana & $R$ & 1 & & 1 & 1 & 1 & & & 4 \\
\hline Tupinambis teguixin & Lobo pollero & $R$ & & & 1 & 1 & & 1 & & 3 \\
\hline Boa constrictor & Boa & R & & & & 1 & 1 & 1 & & 3 \\
\hline Trachemys callirostris & Hicotea & $\mathrm{R}$ & 1 & & 1 & 1 & & 1 & & 4 \\
\hline Rhinoclemmys melanosterna & Galápago & $\mathrm{R}$ & 1 & & 1 & & & & & 2 \\
\hline Caiman crocodylus fuscus & Babilla & $\mathrm{R}$ & 1 & & 1 & 1 & 1 & 1 & & 5 \\
\hline Phalacrocorax olivaceus & Pato yuyo & A & 1 & & & & & & & 1 \\
\hline Ardea herodias & Garza & A & 1 & & & & & & & 1 \\
\hline Ardea cocoi & Garza morena & A & 1 & & & & & & & 1 \\
\hline Chauna chavaria & Chavarrí & A & 1 & 1 & & & & & & 2 \\
\hline Dendrocyna viduata & Viudita & A & 1 & & 1 & 1 & & & & 3 \\
\hline Dendrocygna autumnalis & Pisingo & A & 1 & & 1 & 1 & & & & 3 \\
\hline Cairina moschata & Pato real & A & 1 & 1 & 1 & 1 & & & & 4 \\
\hline Pandion haliatus & Águila pescadora & A & & & & & & & 1 & 1 \\
\hline Busarellus nigricollis & Bebe humo & A & & & & & & & 1 & 1 \\
\hline Buteo magnirostris & Gavilán pollero & A & & & & & & & 1 & 1 \\
\hline Milvago chimachima & Pigua & A & & & & & & & 1 & 1 \\
\hline Ortalis garrula & Guacharaca & A & 1 & & & 1 & & & & 2 \\
\hline Penelope purpurascens & Pavita & A & 1 & & & 1 & & & & 2 \\
\hline Colinus cristatus & Perdiz & A & 1 & & & & & & & 1 \\
\hline Burhinus bistriatus & Galán & A & & 1 & & & & & & 1 \\
\hline Columba cayennensis & Guarumera & A & 1 & & & 1 & & 1 & & 3 \\
\hline Columbina minuta & Tierrerita & A & 1 & & & & & & & 1 \\
\hline Columbina talpacoti & Tortolita & A & 1 & & & & & & & 1 \\
\hline Leptotila verreauxi & Caminera & A & 1 & & & & & & & 1 \\
\hline Ara ararauna & Gonzalo & A & & & & 1 & & & & 1 \\
\hline Aratina pertinax & Cotorra & A & & 1 & & 1 & & & 1 & 3 \\
\hline Brotogeris jugularis & Perico & A & & 1 & & 1 & & & 1 & 3 \\
\hline Amazona ochrocephala & Loro & A & & 1 & & 1 & & & & 2 \\
\hline Ramphastus sulfuratus & Guazalé & A & & 1 & & & & & & 1 \\
\hline Thraupis episcopus & Azulejo & A & & & & & & & 1 & 1 \\
\hline
\end{tabular}




\begin{tabular}{|l|l|l|l|l|l|l|l|l|l|l|}
\hline Volantina jacarina & Mochuelo & $\mathrm{A}$ & & 1 & & & & & & 1 \\
\hline Sphorophilla minuta & Meriño & $\mathrm{A}$ & & 1 & & & & & & 1 \\
\hline Sicalis flaveola & Canario & $\mathrm{A}$ & & 1 & & & & & & 1 \\
\hline Didelphis marsupialis & Zorra chucha & $\mathrm{M}$ & & & & & & 1 & 1 & 2 \\
\hline Dasypus novemcinctus & Armadillo & $\mathrm{M}$ & 1 & & & & & 1 & & 2 \\
\hline Alouatta seniculus & Mono & $\mathrm{M}$ & & & & 1 & & 1 & & 2 \\
\hline Cerdocyon thous & Zorra perro & $\mathrm{M}$ & & & & & & & 1 & 1 \\
\hline Tayassu tajacu & Zaino & $\mathrm{M}$ & 1 & & & 1 & & 1 & & 3 \\
\hline Hydrochaeris hydrochaeris & Ponche & $\mathrm{M}$ & 1 & & & 1 & & 1 & 1 & 4 \\
\hline Agouti paca & Guartinaja & $\mathrm{M}$ & 1 & & & 1 & & 1 & & 4 \\
\hline Dasyprocta punctata & Neque & $\mathrm{M}$ & 1 & & & 1 & & 1 & 1 & 4 \\
\hline
\end{tabular}

Tabla 2. Comportamiento porcentual de los usos detectados de acuerdo con el taxón

\begin{tabular}{|l|c|c|c|c|c|c|c|c|}
\hline Taxón & CCV & MC & HCV & CV & PV & M & D & $\%$ \\
\hline Reptiles & $4(19,1 \%)$ & 0 & $5(23,8 \%)$ & $5(23,8 \%)$ & $3(14,2 \%)$ & $4(19,1 \%)$ & 0 & 100 \\
\hline Aves & $14(31,1 \%)$ & $10(22,2 \%)$ & $3(6,7 \%)$ & $10(22,2 \%)$ & 0 & $1(2,2 \%)$ & $7(15,6 \%)$ & 100 \\
\hline Mamíferos & $5(23,8 \%)$ & 0 & 0 & $5(23,8 \%)$ & 0 & $7(33,3 \%)$ & $4(19,1 \%)$ & 100 \\
\hline
\end{tabular}

Dentro de toda la fauna silvestre analizada los reptiles Caiman crocodilus fuscus, Iguana iguana y Trachemys callirostris poseen un aprovechamiento significativo $(\mathrm{p}<0,05)$, igualmente sucede en aves con Cairina moschata y en mamíferos con Hydrochaeris hydrochaeris, Agouti paca y Dasyprocta punctata; uso que podría ser tildado de altamente preferencial dentro del conjunto general de especies objeto de cacería.

Al comparar entre grupos las aves poseen significativamente $(p<0,05)$ más usos que los reptiles y mamíferos. En cuanto al uso entre taxones no se encontró diferencias significativas $(p>0,05)$ para (CCV) consumo y venta de carne. Se diferencian significativamente $(p<0,05)$ entre taxones (HCV) consumo y venta de huevos y (VP) venta de piel para reptiles, en aves el (MC) uso como mascota, en mamíferos (M) uso medicinal.

Según la Unión Internacional para la Conservación de la Naturaleza UICN (2009) y para Colombia de acuerdo con el INSTITUTO DE INVESTIGACIÓN DE RECURSOS BIOLÓGICOS ALEXANDER VON HUMBOLDT (2008), en general las especies de mayor utilización detectadas en esta investigación: Caiman crocodilus fuscus, Iguana iguana, Cairina moschata, Hydrochaeris hydrochaeris, Agouti paca, y Dasyprocta punctata se tienen clasificadas como en estado de preocupación menor, excepto Trachemys callirostris que se registra como amenazada y vulnerable. 


\section{Discusión}

Al analizar la comunidad rural estudia se puede inferir que los procesos de desarrollo socioeconómico en que están inmersos, se separan ampliamente del bienestar general del Departamento, de la región Caribe y de la región Andina del Colombia; si bien existe acceso a muchos bienes de consumo, el ingreso per capita es bajo y la marginalidad o aislamiento socioeconómico y ambiental es evidente, la utilización de fauna silvestre para comercio y subsistencia, así lo demuestra (DE LA OSSA, 2003; AGUILERA-DÍAZ, 2004; DE LA OSSA y DE LA OSSA-LACAYO, 2011).

El extractivismo de fauna silvestre se puede relacionar con el acceso a productos comerciales, aspecto que tiene que ver con factores tales como: facilidad de acceso a zonas extractivas e ingresos económicos, sin dejar de lado la oferta y aceptación que la fauna tenga en el lugar analizado (DE LA OSSA y DE LA OSSA-LACAYO, 2011). La fauna silvestre cobra mayor importancia en la dieta de poblaciones marginadas rurales, llegando a cubrir un elevado porcentaje de los requerimientos de proteína, como sucede con algunos grupos indígenas y colonos (OJASTI, 1993).

En la extracción de la fauna silvestre, además del aspecto cultural, la facilidad de acceso a la captura durante algunas épocas del año, la ausencia de producción pecuaria alternativa y doméstica menor, el bajo ingreso económico y la marginalidad, hacen que la cacería sea una alternativa viable de subsistencia (REDFORD y ROBINSON, 1991; ALVARD, 2000; HILL y PADWE, 2000; DE LA OSSA, 2003; FUENTES-OBEID et al., 2003; DE LA OSSA y DE LA OSSA-LACAYO, 2011).

En la zona de estudio la utilización de la fauna silvestre muestra un comportamiento similar al registrado para América latina, que posee, igualmente vital importancia social, es así como muchas especies de reptiles, aves y mamíferos mantienen una estrecha relación con la economía de estos grupos humanos, parte de la producción es destinada al consumo y otra parte se usa como bien de cambio (OJASTI, 1993; AQUINO et al., 2001; PERES, 2001; MONROY-VILCHIS et al., 2008). Siendo de gran importancia la obtención de carne para consumo o venta, la cual es significativa y abarca a todos los taxones en este estudio, es además coincidente para la Región como lo demuestran estudios previos (DE LA OSSA y DE LA OSSA-LACAYO, 2011; DE LA OSSA, 2003; FUENTES-OBEID et al., 2003)

Los resultados muestran que no existe una preferencia general por un taxón dado, lo cual puede estar relacionado con: la biomasa, esfuerzo de captura, 
disponibilidad temporal y arraigo en los factores culturales de uso. Sin embargo, de acuerdo al taxón se tiene valor significativo para ciertos usos, lo cual demuestra una clara concepción de los usos y una tendencia comercial especifica. En general, La presión de caza varía dependiendo de la especie, en ecosistemas intactos o preservados los grandes mamíferos están entre los más apreciados, le siguen en su orden aves y reptiles (VICKERS, 1984; REDFORD y ROBINSON, 1991), en estos ecosistemas sujetos a explotación agrícola y pesquera, las aves muestran su importancia al ser el taxón que registra los mayores usos.

Es evidente que la cacería de aves para alimentación, mascotas y venta de crías es relativamente mayor, mientras que para utilización de huevos y pieles los más altos índices de uso se ubican en los reptiles, siendo $C$. crocodilus fuscus, I. iguana y los quelonios los de mayor representatividad, se les utiliza tanto en alimentación como para el comercio de pieles (DE LA OSSA, 2003; DE LA OSSA y DE LA OSSA-LACAYO, 2011). Las aves son relativamente de mayor importancia en la dieta (ALVARD et al., 1997), se tiene un mayor número de especies y su consumo es significativo.

Como lo señalan DE LA OSSA y DE LA OSSA-LACAYO (2011), la utilización generalizada y preferencial de $T$. callirostris (hicotea) hace que la región y su área de influencia sea particularmente característica para este tipo de extracción dentro del territorio nacional, como ya se ha registrado (DE LA OSSA, 2003; FUENTES-OBEID et al., 2003), al igual que lo es para I. iguana (DE LA OSSA, 2003), lo cual en el caso de los quelonios posee una larga y extendida tradición religiosa (PRITCHARD y TREBBAU, 1984; GÓMEZ et al., 2008). No obstante, dada la clasificación CITES (2008) para T. callirostris, su estatus vulnerable la hace una especie a la cual se le debe prestar especial atención y su conservación debe ser prioridad ambiental.

Como indican DE LA OSSA y DE LA OSSA-LACAYO (2011), respecto de los quelonios y de $C$. crocodilus fuscus su uso es comparable con el que se tiene para algunas zonas latinoamericanas de amplios humedales, como la cuenca amazónica, por ejemplo, en donde la oportunidad de acceso y la masiva presencia hacen fácil su captura, además culturalmente tienen una antigua tradición de uso (REDFORD y ROBINSON, 1991; OJASTI, 1993; RODRÍGUEZ y ROJAS-SUAREZ, 1995), como sucede en el municipio de Caimito, en donde además el comercio ilegal ejerce fuerte influencia sobre el extractivismo de estos reptiles todo el año; lo que coincide con lo expresado por DE LA OSSA y DE LA OSSA-LACAYO (2011). 
La venta de crías que se registra para todos los taxones en similaridad proporcional permite inferir que existe un mercado que se mueve alrededor de la compra de neonatos, esta situación genera a mediano plazo un fuerte impacto negativo sobre la renovación poblacional y la estabilidad ecosistémica (DE LA OSSA y DE LA OSSA-LACAYO, 2011). Este tipo de extracción por lo general se orienta hacia el comercio ilegal de especies silvestres, suple la demanda del mercado de mascotas, es sin duda una modalidad de aprovechamiento altamente nocivo (REDFORD y ROBINSON, 1991; OJASTI, 1993; BAPTISTE-BALLERA et al., 2002; DE LA OSSA, 2003), y en nada justifica la subsistencia, dada sus implicaciones ambientales.

Concordando con DE LA OSSA y DE LA OSSA-LACAYO (2011) los resultados obtenidos indican que los pobladores rurales de este estudio, cazan con fines de subsistencia, la alternativa comercial les ofrece la posibilidad de obtener ingresos adicionales que les ayudan a sufragar gastos que también aportan a su diario vivir, aunque normativamente actúen en la ilegalidad. En algunos casos la cacería se orienta a la eliminación selectiva de algunas especies que consideran perjudiciales (REBÊLO y PEZZUTI, 2000), lo cual también hace parte del universo cultural en el que habitan, queda demostrado que solo se aplica a aves y mamíferos, taxones de los cuales algunas especies afectan su escasa producción agrícola, como lo muestran los resultados.

La medicina tradicional posee como característica básica una fuerte y necesaria vinculación entre lo cultural individual y lo social (MOLINA y PEÑALOZA, 2002), específicamente la fauna silvestre también posee vinculación con la medicina tradicional y aunque su uso es menor que el de las plantas, está presente en las comunidades nativas como componente importante (TORRES, 1999; BARBARÁN 2004), así lo muestran los resultados de este trabajo. Se referencia la utilización de ciertas especies con fines curativos, que expresan el uso para este fin del $28,6 \%$ de las especies totales detectadas, siendo principalmente utilizados reptiles y mamíferos, lo que concuerda con lo registrado por DE LA OSSA y DE LA OSSA-LACAYO (2011). Se trata de un valor social importante para la comunidad, comparable con resultados similares a los hallados en México (CORTEZ et al., 2004; ENRÍQUEZ-VÁZQUEZ et al., 2006). Aspecto que no ha sido tenido en cuenta particularmente por la normatividad colombiana, que está poco estudiado en nuestro territorio y que requiere ser abordado científicamente (DE LA OSSA y DE LA OSSA-LACAYO, 2011).

Los efectos de la cacería sobre la fauna silvestre así como sobre los ecosistemas han sido evaluados en diversos países, los cuales muestran cómo se altera la composición faunística y florística, a veces con consecuencias 
drásticas para la diversidad biológica y la subsistencia humana (PERES, 2000), llegando a ser tan graves los efectos en la mayoría de los bosques tropicales que los vertebrados de mayor tamaño desaparecen rápidamente en hábitat relativamente intactos, y esto puede ocasionar el "efecto de bosque vacío" (ROBINSON y BENNETT, 2000); también estos efectos en ciertos casos podrían relacionarse con la alteración de la sinergia existente en el proceso <<fuente-sumidero>> (ROBINSON y BENNETT, 2000; PERES, 2001), lo que en este caso podría estar afectando las poblaciones de mamíferos y reptiles, trasladando la captura mayoritaria hacia las aves (DE LA OSSA y DE LA OSSA-LACAYO, 2011).

Muy a pesar que según los listados oficiales las especies objeto de cacería de este estudio estén clasificadas bajo la denominación de preocupación menor, excepto $T$. callirostris, que se tiene como vulnerable, no se genera la tranquilidad necesaria en asuntos de conservación, porque se desconoce a ciencia cierta el estado poblacional de cada una de ellas para el área de estudio y su gran zona de influencia

\section{Referencias}

AGUILERA-DÍAZ, M. 2004. La Mojana: riqueza natural y potencial económico. Banco de la República. Serie de documentos de trabajo sobre economía regional $N^{\circ}$. 48. Cartagena, Colombia.

AGUILERA-DÍAZ, M. 2005. La economía del departamento de Sucre: ganadería y sector público. Banco de la República. Serie de Documentos de trabajo sobre economía regional $\mathrm{N}^{\circ} 63$. Cartagena, Colombia.

ALDANA, N.J.; DÍAZ-PORRES, M.; FEIJOO, M.A.; ZÚÑIGA, M.C. 2006. Valoración del uso de la fauna silvestre en el municipio de Alcalá, Valle del Cauca. Scientia et Technica XII (31):291-296.

ALVARD, M.; ROBINSON, J.; REDFORD, K.; KAPLAN, H. 1997. The sustainability of subsistence hunting in the Neotropics. Conservation Biology 4:977-982.

ALVARD, M. 2000. The impact of traditional subsistence hunting and trapping populations: data from Wana horticulturalists of Upland Central Sulawesi, Indonesia. Págs. 214-230. En: Robinson, J.G; Redford, K.H. (Eds.). Neotropical Wildlife Use and Conservation. Chicago University Press, Chicago, USA.

AQUINO, R.; PACHECO, T.; VÁSQUEZ, M. 2001.Evaluación y valorización económica de la fauna silvestre en el río Algodón, Amazonía peruana. Revista Peruana de Biología 14(2):187-192. 
BAPTISTE-BALLERA, L.G.; HERNÁNDEZ-PÉREZ, S.; POLANCO-OCHOA, R.; QUICENO-MESA, M.P. 2002. La fauna silvestre colombiana: una historia económica y social de un proceso de marginalización. Disponible en: http://www.humboldt.org.co/pdf/usoyval/Baptiste.pdf. (Consultado: 15-07-2010).

BARBARÁN, F.R. 2004. Usos mágicos, medicinales y rituales de la fauna en la Puna del Noroeste Argentino y sur de Bolivia. Contribuciones al manejo de vida silvestre en Latinoamérica 1:1-26.

CORTEZ, V.; MACEDO, J.; HERNÁNDEZ, M.; ARTEAGA, G.; ESPINOSA, D.; RODRÍGUEZ, J. 2004. Farmacognosia: breve historia de sus orígenes y su relación con las ciencias médicas. Revista Biomédica 15:123-136.

DE LA OSSA, V.J. 1998. Talleres académicos introductorios para la cría y manejo de algunos reptiles. Universidad de Sucre, Facultad de Ciencias Agropecuarias. Sincelejo, Colombia.

DE LA OSSA, V.J. 2003. Manejo de fauna silvestre tropical. Programa de Desarrollo Sostenible de la Región de La Mojana. DNP, FAO, Bogotá, Colombia.

DE LA OSSA, V.J.; DE LA OSSA-LACAYO, A. 2011. Cacería de subsistencia en San Marcos, Sucre, Colombia. Rev. Colombiana cienc. Anim. 3(2):213-224.

EMMONS, L.H. 1991. Neotropical Rainforest Mammals. A Field Guide. The University of Chicago Press. Chicago, USA.

ENRÍQUEZ-VÁZQUEZ, P.; MARIACA-MÉNDEZ, R.; RETANA-GUIASCÓN, O.G.; NARANJO-PIÑERA, E.J. 2006. Uso medicinal de la fauna silvestre en los altos de Chiapas, México. Interciencia 31(7):491-499.

FUENTES-OBEID, S.; SAMPEDRO, M.A.; ARDILA-MARULANDA, M. 2003. Importancia de la jicotea (Trachemys scripta callirostris: Chelonia, Emydidae) como recurso natural en la comunidad de isla del Coco, Región de La Mojana, Departamento de Sucre, Colombia. Revista Biología 17(2):126-133.

GÓMEZ, A.H.; MOLINA, M.; CASTRO, M.; TELLERIA, B.M. 2008. Factores socioecológicos que amenazan a la vida silvestre en la vertiente sur del Parque Nacional Sierra Nevada, Venezuela. Producción Agropecuaria 1(1):40-49.

HILL, K.; PADWE, J. 2000. Sustainability of Aché Hunting in the Mbaracayú Reserve, Paraguay. Págs. 79-105. En: Robinson, J.G.; Bennett, E.L. (Eds.). Hunting for Sustainability in Tropical Forests. Columbia University, New York, USA.

HILTY, S.; BROWN, W. 1986. A Guide of the Birds of Colombia. Princeton University Press. Princeton, USA. 
INSTITUTO DE INVESTIGACIÓN DE RECURSOS BIOLÓGICOS ALEXANDER VON HUMBOLDT. 2008. Catalogo de la Biodiversidad de Colombia. Disponible en: http://Catálogo de la biodiversidad de Colombia. (Consultado 28-02-2012).

MARGOLUIS, R.; SALAFSKY, N. 1998. Measures of success: designing, managing, and monitoring conservation and development projects. Island Press. Washington, DC, USA..

MOLINA, M.; PEÑALOZA, J. 2002. Dog predation on paramo white-tailed deer: the case Mucubaji, Sierra Nevada National Park. Memoria de la Fundación la Salle de Ciencias Naturales 154:139-144.

MONROY-VILCHIS, O.; CABRERA, L.; SUAREZ, P.; ZARCO-GONZÁLEZ, M.M.; RODRÍGUEZ-SOTO, C.; URIOS, V. 2008. Uso tradicional de vertebrados silvestres en la Sierra Nacghititla, México. INCI 33(4):308-313.

OJASTI, J. 1993. Utilización de la fauna silvestre en América Latina, situación y perspectiva para un manejo sostenible. Guía FAO, Conservación № 25, Roma;

OJASTI, J. 2000. Manejo de Fauna Silvestre Neotropical. SIMAB Series N 5. Smithsonian Institution / MAB Program. Washington, D. C, USA.

PÉRES, C.A. 2000. Effects of subsistence hunting on vertebrate community structure in Amazonian forests. Conservation Biology 14 (1):240-253.

PÉRES, C.A. 2001. Synergistic effect of subsistence hunting habitat fragmentation on Amazonian forest vertebrates Conservation Biology 15(6):1490-1505.

PÉREZ, E.M.; OJASTI, J. 1996. La utilización de la fauna silvestre en la América Tropical y recomendaciones para su manejo sustentable en las sabanas. Ecotropicos 9(2):71-82.

POLANCO, R. 2000. Diagnóstico del uso y comercio de fauna silvestre en el Caribe, Pacífico, Andes, Amazonia y Orinoquia colombiana. Instituto de Investigación en Recursos Biológicos Alexander von Humboldt. Bogotá, Colombia.

PRITCHARD, C.P.; TREBBAU, P. 1984. The turtles of Venezuela. Society for the Study of Amphibians and Reptiles. Venezuela.

RAMÍREZ-PERILLA, J. 1996. Tradición de uso y aprovechamiento de fauna silvestre: límites de la sostenibilidad y acciones posibles. Fundación Natura, Organización Regional Indígena Emberá Wounaan-Orewa-Organización de Estados Iberoamericanos para la Educación la Ciencia y la Cultura, Unidad Administrativa Especial del Sistema de Parques Naturales Nacionales del Ministerio del Medio Ambiente. Impreandes Presencia S.A. Bogotá, Colombia. 
REBELO, G.; PEZZUTI, J.B.C. 2000. Percepções sobre o consumo de quelônios na Amazônia. Sustentabilidade e alternativas ao manejo atual. Ambiente \& Sociedade 6/7:85-104.

REDFORD, K.H.; ROBINSON, J.G. 1991. Subsistence and commercial uses of wildlife in Latin America.Págs. 6-23. En: Robinson, J.G.; Redford, K.H. (Eds.). Neotropical wildlife use and conservation. The University of Chicago Press, Chicago, USA.

RENGIFO, J.M.; LUNDBERG, M. 1999. Anfibios y Reptiles de Urrá. Guía de Campo. Skanka. Editorial Colina S.A. Medellín, Colombia.

ROBINSON, J.G.; BENNETT, E.L. 2000. Carrying Capacity Limits to Sustainable Hunting. Págs. 13-30. En: Robinson, J.G.; Bennett, E.L. (Eds.). Sustainability in Tropical Forests. Columbia University Press, N.Y, USA.

RODRÍGUEZ, J.P.; ROJAS-SUAREZ, F. 1995. Libro Rojo de la fauna Venezolana. Provita. Caracas, Venezuela.

STEARMAN, A.M. 2000. A Pound of Flesh: Social Change and Modernization as Factors in Hunting Sustainability Among Neotropical Indigenous Societies. Págs. 233-250. En: Robinson, J.G.; Bennett, E.L. (Eds.). Sustainability in Tropical Forests. Columbia University Press, N.Y, USA.

TORRES, B. 1999.Plantas, curanderos y prospección biológica. Ciencias 5556: 54-60.

UICN, 2009. Biodiversidad. Disponible en: http://www.iucn.org/es/que/biodiversidad/. (Consultado: 28-02-2012).

VICKERS, WT.1984. The faunal components of lowland South American hunting kills. Interciencia 9:366-376. 